\title{
émulations
}

\section{Des ré-actions collectives types}

\section{Déclencheurs types et actions protestataires associées}

\author{
Alessio Motta \\ Émulations - Revue de sciences sociales \\ 2019, $n^{\circ} 31$, «Thomas C. Schelling dans les sciences sociales ».
}

Article disponible à l'adresse suivante

https://ojs.uclouvain.be/index.php/emulations/article/view/motta_schelling

\section{Pour citer cet article}

Alessio Motta, « Des ré-actions collectives types. Déclencheurs types et actions protestataires associées », Émulations, n³1, Mise en ligne le 15 novembre 2019. DOI : 10.14428/emulations.031.04

Distribution électronique : Université catholique de Louvain (Belgique) : ojs.uclouvain.be

(C) Cet article est mis à disposition selon les termes de la Licence Creative Commons Attribution, Pas d'Utilisation Commerciale 4.0 International. http://creativecommons.org/licenses/by-nc/4.0/

Éditeur : Émulations - Revue de sciences sociales / Presses universitaires de Louvain https://ojs.uclouvain.be/index.php/emulations

ISSN électronique : 1784-5734

$\frac{\text { PUL PRESSES }}{\text { UNIVERSITAIRES }}$ 


\title{
Des ré-actions collectives types
}

\section{Déclencheurs types et actions protestataires associées}

\begin{abstract}
Alessio Motta ${ }^{1}$
[Résumé] Cet article s'appuie sur la construction et l'analyse d'une base de données sur 286 mobilisations survenues en France de 1997 à 2000 et relayées dans un quotidien, pour mettre en évidence le rôle de « réactions collectives types » dans le déclenchement d'actions contestataires. Là où les approches en termes de répertoires d'action suggèrent un éventail plus ou moins contraint de choix dans les techniques protestataires disponibles pour un groupe, on mettra en avant l'existence, dans de nombreux cas, d'un mode d'action principal lié à une situation type et qui exerce sur celle-ci une contrainte sans égal : la grève dans des protestations de salariés, la pétition pour certaines mobilisations de quartiers, ou d'autres exemples qui varient au fil de l'histoire et selon les contextes culturels. Cette réaction collective type, point de convergence des attentes des acteurs, s'impose à eux comme une évidence fortement contraignante malgré la diversité de leurs calculs, anticipations, préoccupations et interactions.
\end{abstract}

Mots clés : action collective, action protestataire, déclenchement, mobilisations, répertoires.

\section{Typical Collective Re-actions: Typified Triggers and Associated Protests}

[Abstract] This article is based on the construction and analysis of a database of 286 mobilizations that occurred in France from 1997 to 2000 and were relayed in a daily newspaper. It highlights the role of "typical collective reactions" in the outburst of collective protests. When repertoires of contention theories suggest a more or less constrained range of choice in the protest techniques available to a group, we will emphasize that in many cases there is a main method of action linked to a typical situation and which exerts on it an unparalleled constraint: the strike in the case of employees' protests, the petition for district mobilizations, or other examples that vary over time and cultural contexts. This typical collective reaction, as point of convergence regarding players' expectations, appears to them as strongly binding evidence despite the diversity of their calculations, expectations, concerns, and interactions.

Keywords: collective action, protest, outbreak, trigger, mobilizations, repertoire.

\section{Introduction : déclenchements protestataires et " réactions collectives types ", un panorama}

Cet article enfoncera des portes ouvertes. On y apprendra que pour protester au travail, on fait des grèves, qu'en cas de nuisance dans le quartier, on fait d'abord des pétitions, ou encore que contre l'extrême droite, on manifeste. C'est si évident, pourquoi l'écrire? Parce qu'il s'agira d'enfoncer ces portes avec méthode, de comprendre la portée et les ressorts des liens entre ces situations et types d'actions collectives respectifs. Bref, de les enfoncer à coups d'analyses de données, dans la plus pure tradition scientifique !

\footnotetext{
${ }^{1}$ Université Paris 1 Panthéon-Sorbonne, CESSP, France.
} 
Parmi les thèses les plus courantes pour expliquer ces liens, on trouve celles qui observent que les types de relations (communautaires, associatives, etc.) qu'entretiennent les membres d'un groupe entre eux et avec le reste de la société les orientent vers différentes façons de se mobiliser (Oberschall, 1978 ; Tilly, 1978). Mais ces approches, plus qu'elles n'expliquent le recours à telle technique d'action précise pour tel groupe et dans telle situation, suggèrent une forme de continuité hiérarchisée dans laquelle se classent des groupes plus ou moins organisés, les plus organisés étant supposés avoir un accès plus facile et maîtrisé à l'action protestataire. Les travaux publiés par Charles Tilly à partir des années 1990 ont porté une vision plus détaillée dans laquelle sont distingués, d'une part, les grands répertoires de contestation de l'histoire et, d'autre part, les répertoires tactiques, plus restreints, accessibles à chaque groupe social à un moment donné. Mais que l'on parle des uns ou des autres², l'orientation vers un type de performance dans l'éventail des techniques maîtrisées ou disponibles est toujours présentée, malgré des contraintes diverses, comme un choix des acteurs.

L’idée que je défendrai ici est que cette approche, si elle est pertinente sur la durée d'une mobilisation où les possibles et stratégies en concurrence se diversifient (McAdam, 1983), ne permet pas de rendre compte au mieux de l'étape du déclenchement des premières actions d'une séquence de mobilisations - et donc aussi des nombreuses mobilisations caractérisées par le recours à un seul mode d'action ponctuel. Lorsque l'on s'intéresse aux premières actions décidées et engagées, les contraintes exercées par la situation sont parfois telles que l'idée même d'un choix se révèle discutable. À cette étape, il est souvent plus fécond de voir comment des déclencheurs et situations données imposent restrictivement à des individus l'évidence de réactions collectives types. Ces dernières ne sont pas qu'un jeu de mots. Elles visent à souligner que les scénarios allant de l'absence à l'apparition d'une mobilisation laissent peu de place à l'improvisation, malgré la diversité des calculs et préoccupations individuelles.

Car le lien entre les déclencheurs situationnels et la réaction type est loin d'être mécanique. Il repose sur une infinité de calculs d'anticipation, de négociations et d'autres interactions (dans, avec ou hors des organisations de mobilisations) dans lesquelles émerge la possibilité de recourir à telle ou telle technique d'action collective. Lesdites interactions s'appuient sur des points focaux, c'est-à-dire des points de convergence des attentes des uns sur ce que feront, anticiperont ou accepteront les autres. Thomas Schelling (1986) décrit les points focaux comme des évidences qui, dans des situations de communication limitée, permettent à des acteurs de se coordonner sur une option à choisir ou une action à réaliser en offrant une réponse saillante à la question : à quelle solution penseront les autres, sachant qu'ils sont en train de se poser la même question que moi-même ? Ces évidences, qui contraignent fortement les calculs et négociations des acteurs sur le moment et la façon d'agir, peuvent résulter d'associations d'idées partagées et plus ou moins institutionnalisées. Concrètement, les acteurs reconnaissent

\footnotetext{
2 Ou encore que l'on retienne les usages les plus approximatifs consistant à confondre répertoire et style d'action (Fillieule, 2010 ; Offerlé, 2008 ; Tilly, 2008).
} 
certaines situations ou " déclencheurs types ${ }^{3}$ » (événements, dates clés, repères situationnels divers, etc.) qu'ils savent généralement associés à des types d'actions collectives donnés - éventuellement à des scénarios composés de plusieurs de ces types. Ainsi sont étroitement liés le Premier mai et ses manifestations (Tartakowsky, 2005), les projets et nuisances locales et les pétitions, les bavures policières et les émeutes... Les liens entre déclencheurs et réactions types reposent largement sur des habitudes de groupes ou des stéréotypes largement partagés poussant les acteurs vers des modes d'action donnés, mais la nature de ces liens ne préjuge pas de la façon dont ils font effet. Une réaction collective type, telle un point focal, exerce une emprise forte sur la situation malgré des sens et modes d'appropriation individuels aussi divers que les propriétés et compétences des acteurs, les uns y voyant une évidence naturelle, les autres, plus réflexifs, un cliché qui a la vie dure et dont il faut tenir compte dans leurs calculs.

Cet article ne sera pas consacré à détailler empiriquement la façon dont ces modalités d'appropriation des réactions types construisent la contrainte en situation (et donc le lien causal entre déclencheurs et réactions types) : on renverra sur ce point à un ouvrage plus volumineux (Motta, 2019). Il sera consacré aux réactions types ellesmêmes. Dans une précédente publication (Motta, 2016), j’ai montré que ces associations contraignantes d'idées ne sont pas universelles. Elles valent dans un contexte historique donné, on rejoint sur ce point l'apport fondamental des travaux de Tilly (1978 ; 2008). Chacun de ces liens est le fruit d'un processus d'objectivation puis d'intériorisation de l'association d'idées (dont l'histoire est souvent largement réécrite ou oubliée) et devient une forme d'évidence qui s'impose aux individus (Berger, Luckmann, 2006 [1966]). L'objet de la première publication, « La bavure et l'émeute », était de saisir le processus de construction d'une telle association « déclencheur/réaction collective type » dans l'Est lyonnais. Le présent article est un premier compte-rendu sur la suite de ce travail et son intérêt sera d'autant plus clair que l'on aura lu le précédent. Je chercherai à le compléter en donnant un aperçu de la portée que peut avoir le lien déclencheur/ réaction type sur les actions protestataires en général. Non plus en me concentrant sur la genèse d'un cas particulier, mais par une exploration généraliste de quelques centaines d'actions protestataires survenues en France.

Dans les pages qui suivent, je chercherai à montrer que les réactions collectives types que représentent certains modes d'action traditionnels, notamment dans les mobilisations liées aux mondes du travail, semblent s'imposer avec la plus grande force sur les déclenchements d'actions de protestation. Puis on verra que là où la distinction peut s'opérer, ces modes d'action semblent parfois moins déterminés par les caractéristiques d'un groupe stable qui les maîtriserait que par des circonstances situationnelles. Mais on fera en premier lieu un point sur la méthodologie de l'enquête.

\footnotetext{
${ }^{3}$ Il serait vain de chercher une distinction nette entre ce que j'appelle « déclencheur type » et « situation (déclencheuse) type ». Les deux expressions ont la même signification, si ce n'est que la connotation du mot « déclencheur » me parait plus adéquate pour décrire les cas où cette reconnaissance repose en grande partie sur un événement saillant, et celle du terme « situation » pour les cas où les éléments qui la permettent sont plus composites.
} 


\section{Méthode : constitution et analyse d'une base de données sur des actions protestataires}

La recherche ici présentée est exploratoire, comme son matériau principal. Il s'agit au départ d'un résidu de l'enquête sur les émeutes dans l'Est lyonnais conduite sur la base d’archives de presse des années 1980 et 1990.

\subsection{Constitution d'une base de données à partir des archives du quotidien Le Progrès (1997-2000)}

Pour la dernière période de l'enquête sur l'Est lyonnais (1997-2000), j'ai recouru aux archives numérisées du quotidien Le Progrès, qui offrait des descriptions relativement riches des faits locaux étudiés (Motta, 2016). En plus de la recherche détaillée sur les violences collectives dans la banlieue lyonnaise, j'ai testé par curiosité une recherche rudimentaire sur les publications concernant les actions protestataires en général sur la même période. J'ai ainsi constitué un corpus composé de 598 articles contenant au moins l'une des expressions suivantes : « ont protesté », "protestent » ou « protestaient ».

J'ai lu l'ensemble des articles et relevé les différentes actions de protestation collective qui y sont mentionnées, en excluant les cas situés hors de France, hors de la période étudiée, les informations n'évoquant que des projets d'actions ultérieures comportant une part d'incertitude et les contestations par recours juridiques ou se limitant à la prise de parole d'un ou quelques dirigeants d'organisations. J'ai ainsi constitué une base de données de 286 séquences de mobilisation comportant à chaque fois une ou plusieurs formes d'actions collectives ${ }^{4}$ : grèves, manifestations, pétitions, occupations de locaux, blocages de routes, etc. Pour chacune de ces séquences, j'ai relevé différentes informations, outre les modes d'action utilisés : date de l'article ou des articles ; présence ou non d'organisations politiques, syndicales ou associatives; principaux motifs de mobilisation mis en avant et éléments « supposés » déclencheurs divers (décision affectant les salaires, fermeture d'une classe, nuisance locale...) ; personnes mobilisées (ouvriers, salariés, indépendants, parents d'élèves, habitants d'un quartier, etc.) ; espaces liés à la mobilisation (lieu de travail, établissement scolaire, quartier, etc.) ; contexte national (la mobilisation est-elle locale ou inscrite dans un mouvement plus large). Les modalités n'étaient pas exclusives les unes des autres : à chaque cas pouvaient être attribuées plusieurs modalités pour une même variable. Une mobilisation pouvait par exemple être inscrite à la fois comme liée aux négociations des $35 \mathrm{~h}$ et à l'annonce de suppression de postes dans une entreprise. J'ai également relevé la localisation des faits et distingué ceux ayant eu lieu dans l'ancienne région Rhône-Alpes (186 cas) des autres (100 cas), afin d'observer si les cas éparpillés dans l'hexagone faisaient l'objet d'une sélection et d'un traitement fortement différents de ceux couverts dans le cadre de l'activité journalistique régionale. La comparaison des bases de données incluant ou

\footnotetext{
${ }^{4}$ Cette liste aurait bien entendu pu être étendue à d'autres éléments susceptibles de jouer sur le mode d'action, comme les cibles (Martin, McCarthy, Walker, 2008).
} 
excluant les faits ayant eu lieu hors de la région Rhône-Alpes n’a pas mené à observer de différences majeures. J'ai donc choisi de conserver la base entière, pour travailler sur un effectif plus important.

Le nombre de 286 mobilisations ne correspond pas précisément au nombre d'articles décrivant des actions contestataires : certaines actions sont décrites par plusieurs articles et certains articles décrivent plusieurs actions. J'ai cherché à réunir pour chaque ligne de la base de données des séquences d'action contestataires s'étalant sur un ou quelques jours. Mais une même mobilisation revenait sur plusieurs lignes si elle s'étalait sur une ou plusieurs semaines et faisait l'objet d'articles multiples. Un mouvement traité en des termes unifiés mais dont un article décrit un exemple d'action à Besançon et un autre à Lyon faisait l'objet de deux lignes différentes dans la base de données, à condition que les exemples cités donnent lieu à des descriptions relativement détaillées et ne se limitent pas à l'énumération d'une liste de villes.

\subsection{Limites et intérêt de l'analyse de données issues d'un quotidien}

Reste que, tant dans la constitution de ces 286 unités que dans les catégories utilisées pour décrire les situations de mobilisations, nombre de cas limites ont donné lieu à des choix qui comportent une part d'arbitraire. Les termes inscrits dans le moteur de recherche, s'ils visent à trouver des articles évoquant des actions protestataires diverses, sont eux-mêmes probablement générateurs de biais.

Pas autant, d'ailleurs, que le fait d'étudier les actions protestataires en consultant les informations qui ont été sélectionnées et mises en forme à travers le processus de production journalistique. Le choix du matériau génère inévitablement certains des biais soulignés entre autres par les critiques de la protest event analysis (Fillieule, 2008). On n'échappera par exemple pas au fait que les mobilisations relayées dans la presse, même régionale, ne constituent qu'un échantillon des mobilisations qui ont lieu. $\mathrm{Ni}$ aux effets des cycles d'attention médiatique dont j'ai pu ressentir l'impact au moment de la constitution de la base de données, par exemple dans le traitement manifestement inégal d'une année à l'autre des mobilisations locales de parents qui émaillent chaque rentrée scolaire. Ni encore aux diverses forces qui président à la sélection et à la production de l'information, parfois aussi contradictoires que peuvent l'être la recherche de confirmation de clichés et la quête de l'anecdote originale. Ni enfin au biais finaliste résultant du fait que je ne traite que de cas où des actions collectives ont effectivement eu lieu.

Les résultats de cette enquête paraissent malgré tout dignes d'être exploités pour différentes raisons. Tout d'abord, le recours à un journal régional limite les effets des cycles d'attention médiatique nationaux en cédant une place aux logiques de travail des localiers. Ensuite, cette recherche ne vise pas à la comparaison internationale ou historique. Elle n'est donc pas affectée par les défauts liés à la dimension comparatiste de la protest event analysis (commente-t-on des différences dans les mobilisations ou dans leurs traitements médiatiques ?). Par ailleurs, elle est loin d'être une fin en soi : elle 
s'articule avec des recherches qualitatives comme celles mentionnées en introduction. Enfin, les statistiques présentées plus bas n'ont tout simplement pas vocation à être interprétées comme représentatives de ce que sont les mobilisations en France. Elles visent à donner un ordre d'idée de la force des liens qu'entretiennent certaines situations types avec certains types d'actions protestataires. Du moins sur une période et un territoire donnés, ces liens n'étant ni universels, ni figés.

La première étape du travail d'exploitation de la base de données a été la réalisation d'une analyse de correspondances multiples (ACM). Cette méthode d'analyse factorielle vise à représenter sur un graphique les corrélations entre les diverses modalités de la base, en accordant un poids (taux de contribution aux axes) variable à chacune d'elles en fonction de sa fréquence et de la force de ses liens avec d'autres. L'ACM servira moins à représenter un espace concret qu'à faire un repérage exploratoire des liens respectifs entre types d'acteurs, situations et mobilisations, qui sera affiné ensuite par des tris croisés.

\section{Des traditions qui s'imposent : la force contraignante de modes d'action traditionnels sur les déclenchements}

On fera un premier tour d'horizon des associations qui se dégagent de l'ACM avant de se concentrer sur les exemples liés aux mobilisations de salariés. 


\subsection{Premier tour d'horizon}

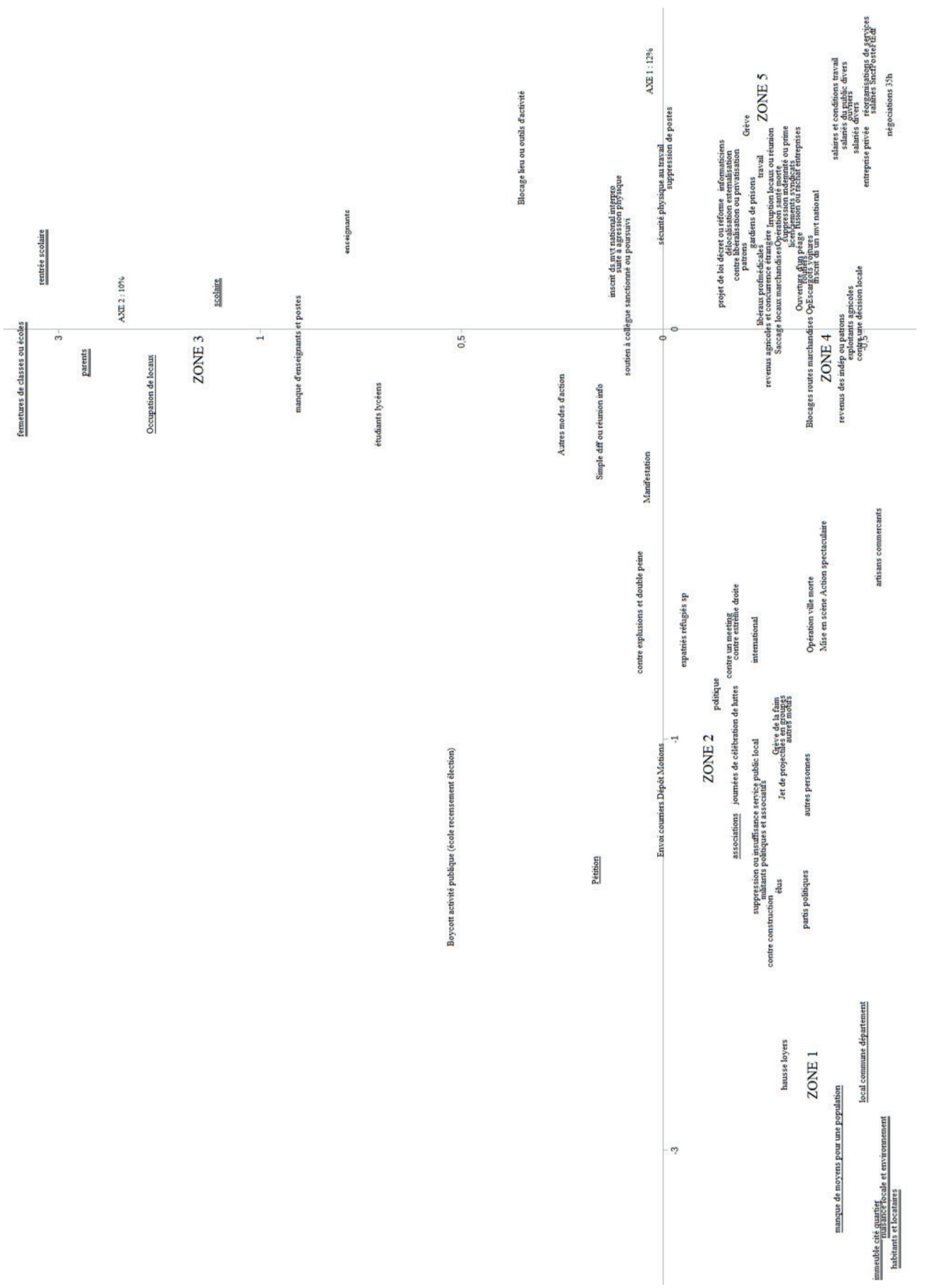

FigURE $N^{\circ} 1$ : ANALYSE DES CORRESPONDANCES MULTIPLES (ACM) SUR LES ACTIONS PROTESTATAIRES ISSUES DE LA RECHERCHE DANS LE PROGRĖS (1997-2000) 
L'ACM compte 44 modalités actives dont 5 ayant des taux de contribution à un des axes allant de 5 à $10 \%$ (soulignées) et 7 ayant des taux supérieurs à $10 \%$ (doublement soulignés ${ }^{5}$ ). Les positions des modalités soulignées ne laissent pas de mystère sur l'axe auquel chacune contribue fortement. Les disproportions d'affichage causées par la présence de fortes contributions en haut et à gauche ne doivent pas conduire à ignorer les caractéristiques globales du graphique. Par exemple, le fait que le positionnement des modalités sur l'axe 1 est largement déterminé par leur relation ou non avec une activité professionnelle, ou la concentration en bas à droite d'assez nombreuses modalités caractérisées par des petits taux de contribution mais parmi lesquelles se dégagent plusieurs zones. Sur l'ensemble du graphique, on distinguera cinq zones principales.

La zone 1, à gauche, est celle des mobilisations locales ou territoriales. On y trouve les actions protestataires menées à l'échelle d'immeubles, quartiers, communes et départements, comme celles liées à la construction ou à la présence de structures générant des nuisances pour l'environnement et la population locale. Les principaux modes d'action utilisés dans ces cas sont la pétition et la manifestation, on y reviendra plus loin. Ce n'est pas manifeste sur le graphique puisque ces techniques, en particulier la manifestation, ne sont pas spécifiques aux mobilisations locales. La zone 2, au centre gauche, est principalement celle des mobilisations de militants et acteurs politiques ou associatifs. On y trouve entre autres des actions contre l'extrême droite ou pour des causes internationales. Le principal mode d'action associé à cette zone, d'après les tris croisés, est la manifestation. La zone 3, en haut, est celle des mobilisations en milieux scolaires, avec en périphérie les actions de parents d'élèves contre les fermetures de classes, qui consistent fréquemment en des occupations de salles de classe. Chez les étudiants et lycéens, la modalité d'action la plus souvent enregistrée est la manifestation. Chez les enseignants, la grève. La zone 4, en bas, est celle des mobilisations d'artisans, commerçants, indépendants et agriculteurs. Ces groupes sont tous associés à une relative diversité de modes d'action : manifestations, mises en scène, saccages de locaux ou marchandises, blocages de routes ou de marchandises, etc. Enfin, la zone 5, en bas à droite, est celle des mobilisations de salariés. Là encore, les tris montrent la présence de nombre de manifestations. Mais le mode d'action roi y est la grève.

\subsection{Salariés, grève et autres modes d'action : des traditions bien établies}

Les mobilisations liées à l'activité professionnelle, en particulier celles des salariés, forment certainement l'ensemble le plus riche d'enseignement sur la force des réactions collectives types. Elles couvrent respectivement les deux tiers et la moitié de l'échantillon et sont marquées par de fortes régularités. Là où l'importante présence syndicale

\footnotetext{
${ }^{5}$ L'ACM a été réalisée sur LibreOffice Calc avec Lenuagepourtous. J'ai défini toutes les variables comme a priori actives et fixé le seuil d'activation d'une modalité à $3 \%$ de l'effectif total, soit 9 occurrences au moins. Les modalités présentes dans 8 cas ou moins sont illustratives. Pour améliorer la lisibilité, j'ai appliqué un coefficient de dilatation de 1,5 aux coordonnées des modalités illustratives puis, comme le montre la graduation, dilaté l'affichage du centre du graphique (coordonnées situées entre -1 et 1 ).
} 
(mentionnée dans 75 à $100 \%$ des cas dans les grandes catégories de salariés) pourrait apparaître comme un facteur d'enrichissement des possibilités de mobilisation, elle se présente statistiquement comme une contrainte. Pour les principales catégories de salariés, les articles mentionnent un recours à la grève dans 86 à 91 \% des cas ${ }^{6}$.

Tableau $\mathrm{N}^{\circ} 1$ : Part des cas mentionnant un recours À la grève DANS LES PRINCIPALES CATÉGORIES DE SALARIÉS

\begin{tabular}{|l|c|c|}
\hline Catégories de salariés & $\begin{array}{c}\text { Cas mentionnant une grève } \\
(\%)\end{array}$ & $\begin{array}{c}\text { Cas mentionnant une grève } \\
\text { (valeurs absolues) }\end{array}$ \\
\hline Ouvriers & $91 \%$ & 20 sur 22 \\
\hline $\begin{array}{l}\text { Salariés Sncf, Poste, France } \\
\text { Tél., EDF }\end{array}$ & $87 \%$ & 20 sur 23 \\
\hline Salariés du public divers & $86 \%$ & 42 sur 49 \\
\hline $\begin{array}{l}\text { Salariés autres (privé, } \\
\text { divers) }\end{array}$ & $86 \%$ & 18 sur 21 \\
\hline Enseignants & $73 \%$ & 22 sur 30 \\
\hline
\end{tabular}

La manifestation est fréquemment présente, mais dans des proportions moindres : 15 à $40 \%$ des cas selon les catégories. Les divers autres modes d'action sont bien rares : sur l'ensemble de ces catégories, $79 \%$ des articles ne mentionnent rien d'autre que des grèves et/ou des manifestations. Encore une fois, ces taux ne sont pas tous représentatifs de la réalité des mobilisations, ne serait-ce que parce que le travail journalistique tend à effacer certains modes d'action quand d'autres, plus visibles, sont présents. Les distributions de tracts ou de pétitions ne sont par exemple que rarement mentionnées quand elles ont lieu lors d'une manifestation ou d'une grève, alors qu'elles y sont souvent présentes.

Reste que peu de catégories semblent recourir à un répertoire si étroit que celui des salariés, surtout si l'on se focalise davantage sur la question qui est au fondement de cette recherche, à savoir le déclenchement des séquences d'actions collectives. La plupart des articles évoquant une action protestataire ne précisent pas si celle-ci est isolée ou si d'autres modes d'action ont été utilisés dans les heures ou jours qui précèdent. Cependant, j'ai isolé 39 articles évoquant une séquence de plusieurs modes d'action successifs et/ou simultanés, par lesquels il est possible de reconstituer de façon au moins rudimentaire l'ordre des événements et, dans la plupart des cas, d'avoir une idée des premières techniques d'actions utilisées 7 . Parmi ces articles, 20 concernent les catégories de salariés mentionnées ci-dessus. Dans 18 cas, soit 90 \% des cas, la grève est

\footnotetext{
${ }^{6}$ Reprenant les usages habituels du terme et ceux rencontrés dans les articles, j'ai désigné par " grève » un mode d'action consistant à cesser le travail, au sens large pour éviter de réserver tautologiquement la catégorie aux salariés. J'y ai inclus les grèves étudiantes, assez rares dans le corpus, les « grèves administratives » de pompiers et « grèves tournantes » des personnels hospitaliers.

${ }^{7}$ Bien que tous les cas n'apportent pas de garanties de l'absence d'autres modes d'action encore antérieurs. Notons que sont exclus de ces 39 articles les cas courants où sont « seulement » organisées une grève et une manifestation le même jour.
} 
la première action décidée ou réalisée, seule ou accompagnée d'autres actions simultanées.

Une autre approche permettant de saisir la contrainte exercée par la grève sur les déclenchements d'actions protestataires de salariés consiste à s'intéresser à ceux qui y échappent. Car force est de constater que les salariés qui se mobilisent autrement n'ont pas pour point commun un plus large éventail de possibilités, mais plutôt des contraintes propres au moins aussi fortes. Ces contraintes sont de deux ordres. Elles sont d'abord celles des groupes qui, pour des raisons juridiques ou techniques, ne peuvent se mettre en grève. C'est le cas des gardiens de prison, rencontrés à six reprises dans le corpus, qui contournent l'interdiction en bloquant l'entrée de l'établissement, en prenant des jours de congé et, dans deux cas, en construisant un mur symbolique mais cimenté. Elles sont ensuite celles des groupes auxquels est associé un mode d'action type qui leur est propre et très fortement objectivé par l'histoire. On pensera au cas des routiers, catégorie indigène lourde d'effets, menant régulièrement à confondre des salariés, artisans et patrons dans une même catégorie et posant sur les épaules de ces acteurs le poids d'une histoire (Courty, 1993) qui les conduit à partager la technologie d'action du blocage des routes dans les cinq cas rencontrés dans le corpus (avec certes quelques différences dans les lieux et techniques d'application). Un cas emblématique est celui des mouvements de fin 1999 et début 2000 où les patrons routiers organisent des blocages et obtiennent des dérogations à la loi sur les 35h, auxquelles les organisations de salariés s'opposent à leur tour par des blocages (Le Progrès, 24 décembre 1999, 12 janvier 2000 et $1^{\text {er }}$ février 2000).

La grève comme le blocage routier se présentent donc à leurs utilisateurs respectifs moins comme des possibilités parmi d'autres dans leurs paniers que comme des techniques quasi-incontournables au moment de se lancer dans des actions de protestation collective. Néanmoins, les réactions types sont loin d'être toujours associées aux caractéristiques d'un groupe.

\section{Groupes de circonstances, situations et déclencheurs types : une primauté des données situationnelles dans la détermination du type d'action déclenché ?}

Par primauté, j’entends le fait que, dans la majorité des cas, les données situationnelles semblent être celles qui offrent les corrélations les plus fortes avec des modes d'action donnés, soit en resserrant les possibilités offertes par le répertoire d'un groupe relativement stable, soit en orientant l'action de groupes formés par les circonstances. 


\subsection{Les situations et déclencheurs types, goulots d'étranglement des répertoires tactiques}

Les mobilisations de salariés offrant des taux de corrélation avec le recours à la grève extrêmement élevés, on retrouve sans surprise des taux similaires, de l'ordre de 80 ou $90 \%$ lorsque l'on s'intéresse aux situations ou motifs supposés générateurs d'action collective. J'ai fait le choix, dans le traitement des données, de ne pas opérer de distinction nette entre situations et motifs. C'est sans doute donner aux motifs un poids plus important que celui qu'ils représentent réellement dans la détermination du passage à l'action (Motta, 2016 ; Snow, Zurcher, Ekland-Olson, 1980). Mais le contraire aurait conduit à des classements absurdes, les situations générant des mobilisations devenant souvent des motifs mis en avant par les acteurs mobilisés, qu'il s'agisse de suppressions de postes, de réorganisations de services dans l'entreprise ou d'insécurité physique. Les exemples du tableau 2 en donnent un aperçu, les cas les plus fortement associés à la grève sont ceux où des décisions ou circonstances affectent directement, récemment ou dans un avenir proche, les salariés d'une unité réduite (usine, service d'une entreprise, établissement scolaire, etc.). Les questions liées aux agressions et risques physiques entrainent même systématiquement des grèves, à l'exception d'un cas où il s'agit de gardiens de prison.

Tableau N $^{\circ} 2$ : Part des cas mentionnant une grève Selon des Situations ET MOTIFS LIÉS AU TRAVAIL SALARIÉ

\begin{tabular}{|l|c|c|}
\hline $\begin{array}{l}\text { Situations et/ou principaux } \\
\text { motifs relevés }\end{array}$ & $\begin{array}{c}\text { Cas mentionnant une grève } \\
(\%)\end{array}$ & $\begin{array}{c}\text { Cas mentionnant une grève } \\
\text { (valeurs absolues) }\end{array}$ \\
\hline $\begin{array}{l}\text { Salaires et conditions de } \\
\text { travail }\end{array}$ & $82 \%$ & 82 sur 100 \\
\hline Négociations liées aux 35h & $83 \%$ & 19 sur 23 \\
\hline Suppression de postes & $89 \%$ & 16 sur 18 \\
\hline Réorganisation de services & $90 \%$ & 18 sur 20 \\
\hline $\begin{array}{l}\text { Agression + sécurité phys. } \\
\text { au travail }\end{array}$ & $91 \%$ & 10 sur 11 \\
\hline
\end{tabular}

L’importance des situations et déclencheurs types est plus visible si l'on se déplace vers la zone 3 de l'ACM qui, plaçant en périphérie les « fermetures de classes » et la " rentrée scolaire », suggère que ces modalités sont associées à des corrélations plus fortes que les types de groupes mobilisés. C’est le cas notamment si l'on s'intéresse aux occupations de locaux, mode d'action qui, sans être spécifique au milieu scolaire, y est fréquemment rencontré (figure $\mathrm{n}^{\circ} 2$ ). Les occupations de classes, en l'occurrence, concernent un peu moins de la moitié des cas de mobilisations de parents d'élèves relevés dans le corpus. Bien que l'on parle ici de petits nombres, il convient de relever qu'elles sont plus fortement associées à certaines situations précises : les fermetures de classes et périodes de rentrée scolaire. Le croisement de la modalité « rentrée scolaire » 
(mobilisations en milieu scolaire dans les sept premiers jours de septembre) avec l'une ou l'autre des précédentes conduit à des taux de recours à l'occupation de classes supérieurs à $80 \%$.

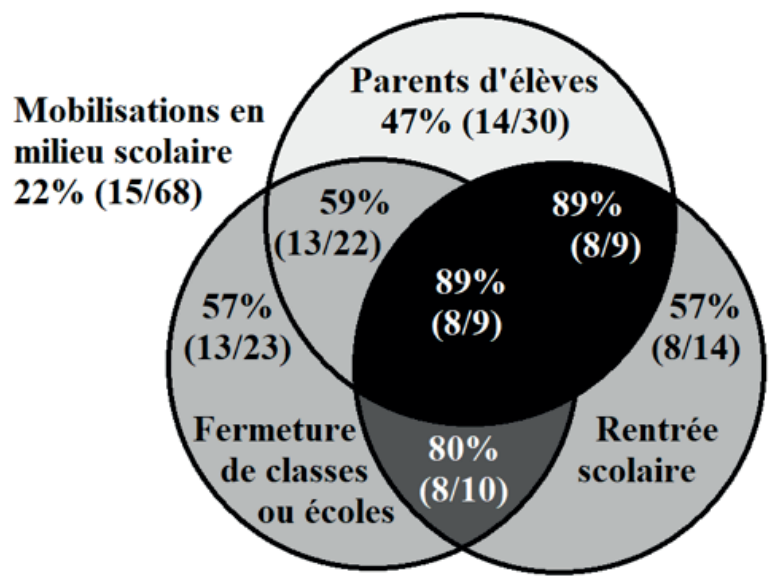

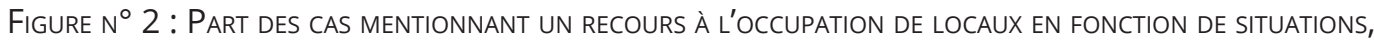
MOTIFS ET PARTICIPANTS CONCERNÉS EN MILIEU SCOLAIRE, EN \% (ET VALEURS ABSOLUES) ${ }^{8}$

Le croisement des modalités agit ici comme une cumulation de propriétés déterminantes dans laquelle les données situationnelles jouent un rôle relativement plus important que celles qui caractérisent les personnes mobilisées. Plus important car elles sont associées à des corrélations plus fortes et parce qu'elles amènent d'autres personnes que les parents d'élèves à participer (certains articles mentionnent la participation d'enseignants à ces occupations). Mais, plus précisément, elles semblent quasiment réduire le répertoire relativement étendu observé chez les parents d'élèves (manifestation, pétition, boycott, etc.) à un seul mode d'action. Il est tentant de voir dans l'occupation de salles de classe un mode d'action qui serait une réponse tactique logique pour des jours de rentrée scolaire. Mais se contenter de cette explication serait ignorer les effets de la médiatisation nationale et locale de ce type de réaction collective à court et moyen terme. Lors de certaines rentrées, les occupations de classe sont décrites, en l'espace de quelques jours, comme une vague relativement dense de réaction aux fermetures de classes (en particulier en 1999) qui rend la technique non seulement pensable, mais même évidente en contexte similaire. Il est tentant aussi d'attribuer le poids de cette technique dans le corpus à sa capacité à attirer l'attention médiatique. L'argument est au moins en partie fondé si on oppose l'occupation de classe à la pétition : hors des périodes de rentrée scolaire, on relève quatre pétitions de parents d'élèves, toutes situées dans la région Rhône-Alpes, autrement dit, de telles pétitions semblent difficilement pouvoir traverser le processus de production journalistique

\footnotetext{
${ }^{8}$ Les données situées hors du croisement entre deux ou trois cercles concernent l'ensemble des cercles dans lequel elles s'inscrivent. Par exemple, les 14/30 évoqués en haut représentent l'ensemble des cas impliquant des parents d'élèves, qu'ils soient ou non liés à des fermetures ou à la période de rentrée scolaire. Ces remarques valent pour les graphiques suivants.
} 
hors des pages locales et se voient forcément sous-représentées face à un mode d'action dont les journalistes relaient également les occurrences situées dans le reste du pays. Mais les manifestations de parents contre les fermetures de classes ne semblent pas subir ce handicap et, hors périodes de rentrées scolaires, on en trouve cinq dans le corpus, pour cinq occupations de classes liées aux mêmes types de motifs, parfois sur des laps de temps resserrés. Il y a donc fort à parier que la très forte domination de la technique de l'occupation de classes sur les périodes de rentrées soit une réalité et non un artefact d'enquête. Ce constat ne préjuge bien sûr pas d'une unique façon d'opter pour cette technique. Comme pour tous les cas évoqués dans l'article, une réaction collective type ne s'applique pas mécaniquement (Motta, 2019) : elle pèse sur les calculs et interactions lors desquelles émergent les choix d'action disponibles au moment de la rentrée. Calculs et interactions qui peuvent prendre des formes diverses selon les établissements, caractéristiques des acteurs et la présence ou non d'associations de parents d'élèves.

L'effet cumulatif observé autour des occupations de salles de classe se retrouve dans d'autres cas comme celui des manifestations étudiantes. Sur 16 cas de mobilisations étudiantes ou lycéennes, 13 (soit $81 \%$ ) passent par des manifestations (dans certains cas, des blocages sont également organisés les mêmes jours). Et cela qu'il s'agisse de mobilisations s'inscrivant dans un mouvement national ou de situations très locales regroupant un petit nombre d'étudiants (dans un institut de formation, par exemple), si bien que le recours à la manifestation ne semble pas lié au nombre de participants potentiels. Sur les 16 cas, 13 sont liés à des situations et motifs relativement courants pour de tels mouvements : manque d'enseignants et de postes, projet de réforme, hausse des frais d'inscription dans un établissement en particulier, etc. Or, sur ces 13 cas, 12 (soit $92 \%$ ) passent par des manifestations. Sur les trois cas de mobilisations d'étudiants ou lycéens où aucune manifestation de rue n'est évoquée, deux sont liés à des motifs assez singuliers : l'admission des garçons dans une cité étudiante jusque-là réservée aux filles, qui donne lieu à une pétition (Le Progrès, 23 février 1997), et l'enlèvement d'un préau et d'un abribus à proximité du lycée, suivi d'une « grève » des élèves (Le Progrès, 7 octobre 1998). Là où les situations et déclencheurs couramment associés aux étudiants et lycéens tendent à restreindre leur répertoire à son mode d'action roi? ce sont donc les situations plutôt singulières qui ouvrent la porte à des modes d'action plus singuliers, ou en rapport avec les caractéristiques situationnelles. Le cas de la cité pour filles évoque bien entendu les mobilisations d'habitants d'immeubles qui, ellesmêmes, débutent le plus souvent par des pétitions.

\footnotetext{
${ }^{9}$ La méthode employée ici invisibilise cependant une spécificité des mouvements étudiants, à savoir le recours préalable aux assemblées générales, technique moins clairement typifiée comme contestataire en soi, mais qui s'intègre quasi-systématiquement aux scénarios de contestations collectives (Le Mazier, 2015).
} 


\subsection{La situation comme déterminant principal du mode d'action et le cas des mobilisations locales}

Les deux modes d'action qui ressortent nettement des mobilisations locales sont les pétitions et les manifestations. Si l'on s'intéresse aux deux modalités les plus nombreuses dans ces mobilisations, " habitants et locataires » et " nuisance locale et environnement ${ }^{10}$ ", aucun de ces deux modes d'action ne ressort plus que l'autre. Le type d'espace concerné par les motifs de mobilisation joue cependant un rôle déterminant, avec un effet encore une fois cumulatif lorsque sont présentes les modalités les plus fortement liées aux mobilisations locales. Le type d'espace, ou plutôt, en l'occurrence, l'échelle de cet espace : les actions de très petite échelle (immeuble, cité, quartier) ramènent dans l'essentiel des cas à des pétitions (figure $\mathrm{n}^{\circ} 3$ ) et celles liées à un échelon allant d'une commune à un département, aux manifestations (figure $\mathrm{n}^{\circ} 4$ ).

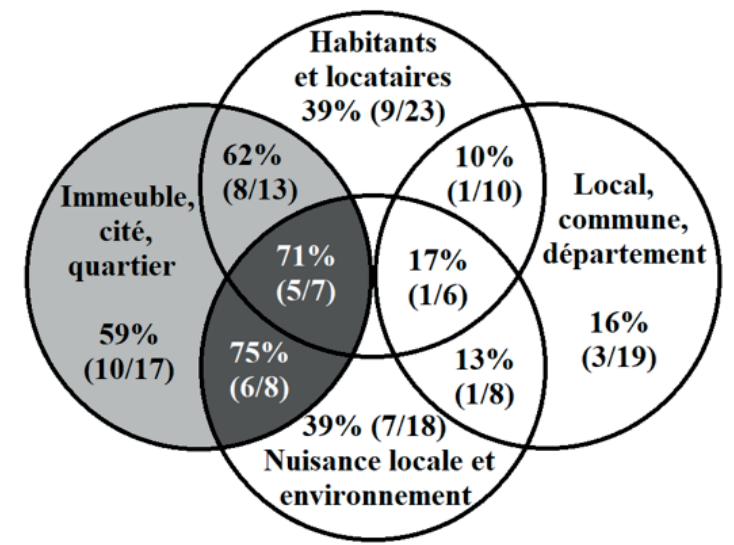

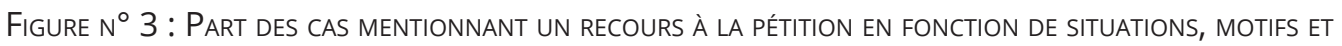
PARTICIPANTS CONCERNÉS DANS LES MOBILISATIONS LOCALES, EN \% (ET VALEURS ABSOLUES)

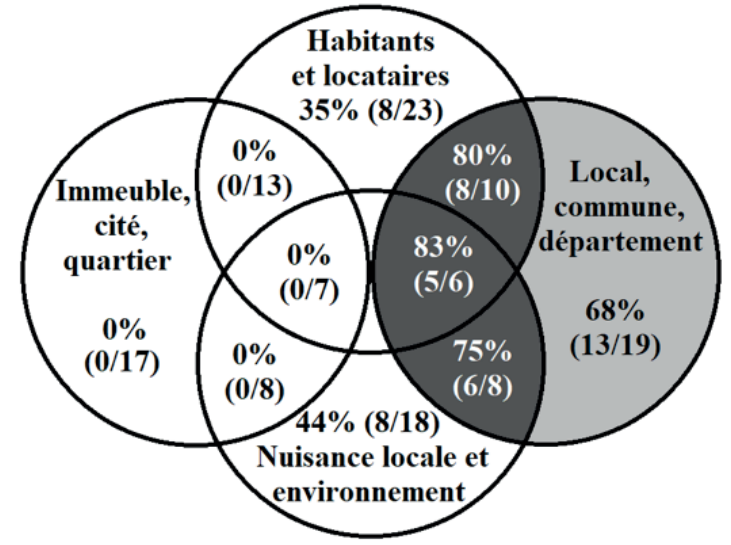

FIGURE N ${ }^{\circ} 4$ : PART DES CAS MENTIONNANT UN RECOURS À LA MANIFESTATION EN FONCTION DE SITUATIONS, MOTIFS ET PARTICIPANTS CONCERNÉS DANS LES MOBILISATIONS LOCALES, EN \% (ET VALEURS ABSOLUES)

\footnotetext{
${ }^{10}$ La catégorie peut sembler étrange, mais toutes mobilisations liées à des causes environnementales du corpus étaient aussi liées à une nuisance ou un projet local en particulier (projet de centre d'enfouissement de déchets nucléaires, d'extension d'un aéroport, etc.). Il y a fort à parier qu'un corpus d'articles plus récents aurait donné un résultat différent.
} 
Ici encore, il semble que les réactions types restreignent d'autant plus fortement les marges de manœuvre concernant les modes d'action disponibles que l'on se trouve dans des situations types où les acteurs et groupes, espaces concernés, motifs et autres éléments circonstanciels forment une association cohérente vis-à-vis des clichés sur les mobilisations (habitants d'un quartier souhaitant agir contre une nuisance locale, etc.). Ces marges de manœuvre sont au contraire plus importantes lorsque le mélange des modalités apparaît singulier ou original.

De façon générale, la contrainte exercée par les réactions collectives types apparaît moins liée à des groupes constitués et dotés d'un répertoire donné qu'à des attentes et à des évidences liées à des situations. C'est en tout cas ce que laisse penser le fait que les mobilisations concernant les habitants d'une zone donnée, souvent constitués en groupe par des circonstances récentes, passent par des pétitions ou des manifestations en fonction de l'échelle. D'autant que la présence d'associations ou d'organisations menant une activité durable autour des motifs de mobilisation n'a pas d'influence particulière sur les techniques d'action mobilisées : sur les deux cas où elle est mentionnée pour des mobilisations d'immeubles, cités ou quartiers, un passe par une pétition - à peu près le même taux qu'en l'absence de cette mention - et sur les huit cas pour les mobilisations de niveau communal à départemental, sept passent par des manifestations. Ici encore, il est tentant d'attribuer le choix d'un mode d'action à des considérations tactiques résultant directement de l'échelle de mobilisation : la manifestation exigerait d'être plus nombreux. S'il est vraisemblable que ce type d'argument soit présent dans les discussions où se joue le choix du mode d'action, on ne saurait s'en contenter, notamment au vu de l'existence dans les mobilisations en question de plusieurs manifestations de très petite taille (moins d'une vingtaine de participants) parvenues à se frayer un chemin dans les pages du quotidien.

En outre, si l'on se concentre sur les cas où une chronologie de modes d'action multiples ressort des articles, les quelques données disponibles confirment que dans tous les cas où le mode de réaction type mis en évidence plus haut est présent (un cas de pétition pour les immeubles, cités, quartiers et trois cas de manifestations pour le local, commune, département), il est mis en œuvre en premier, que ce soit seul où simultanément à d'autres. Globalement, les 19 articles laissant entrevoir une telle chronologie et ne concernant pas des salariés confirment encore que les modes de réactions types associées à telles catégories ou circonstances sont ceux qui sont utilisés en premiers. Si l'on ajoute cela aux corrélations particulièrement fortes entre données situationnelles et mode d'action présentées plus haut, tout se passe comme si, au-delà des répertoires d'action liés à tel groupe et des calculs tactiques, le fait d'opter pour tel ou tel mode d'action était surtout le fruit d'évidences imposées par des situations. À travers, par exemple, un réflexe qui consiste à voir la pétition comme un moyen logique et naturel pour entamer une mobilisation concernant un petit espace d'habitants, que les rationalisations qui la soutiennent portent sur son caractère simple et " évident » ou sur l'ambivalence d'un mode d'action qui est à la fois moyen de diffusion de la lutte et 
" test " sur sa capacité à récolter des soutiens relativement peu coûteux (Contamin, 2009 ; Motta, 2014 ; Rémy, 1995).

Ces évidences peuvent cependant résulter de typifications et d'histoires dans lesquelles les groupes habituellement mobilisés ont un rôle de poids. Ainsi, le lien entre les mobilisations communales ou départementales et les manifestations ne saurait être pensé indépendamment de la porosité de ces échelons d'actions vis-à-vis des organisations politiques. En témoigne, outre le positionnement de ces dernières dans l'ACM (figure $\mathrm{n}^{\circ} 1$ ), le fait que sur les 19 cas concernant l'échelon en question, quatre mentionnent les soutiens ou présences d'élus et trois autres la présence de partis politiques. Or, le lien entre les mobilisations directement liées au champ politique et les manifestations est l'un des plus marqués dans le corpus : la quasi-totalité des cas où sont présents des élus et militants politiques ou des motifs tels que l'opposition à l'extrême droite ou à un meeting donnent lieu à des manifestations.

\section{Conclusion : poids d'une contrainte située et situationnelle}

Dans les pages qui précèdent, j'ai cherché à mettre en avant la fréquence des cas où l'action protestataire consiste en une réaction collective type. C'est-à-dire en un mode d'action associé à un type donné de situation qui se présente aux acteurs, dans leurs propositions et négociations, non comme une possibilité parmi d'autres dans un répertoire mais comme un point focal, une évidence vers laquelle convergent les anticipations réciproques, du moins lorsqu'il s'agit de se lancer dans une séquence de mobilisation. Cette évidence repose parfois sur l'accumulation et la sédimentation d'histoires de luttes ou sur la perception d'une continuité entre la situation locale et celle d'autres sites dans lesquels s'est déjà engagé un mouvement similaire dont on reprend les outils (McAdam, 1995). Mais elle peut aussi reposer sur des clichés, souvent assez largement partagés pour produire des effets sur des groupes formés d'individus qui, comme les parents d'élèves ou les habitants de tel immeuble, sont loin d'être impliqués de manière régulière dans des mobilisations collectives. Ces clichés sont associés à des catégories hétérogènes et arbitraires (groupe statutaire ou corporatif, cadre temporel ou géographique de la protestation, motifs, etc.) car ils sont le fruit d'histoires de luttes largement singulières et non d'une logique globale et cohérente. Si la construction des souvenirs de mobilisations " réussies » peut jouer un rôle clé dans ces histoires, les réactions types représentent le plus souvent des obstacles aux calculs sur les modes d'action les plus « efficaces », que ce soit sur le plan de la facilité et du volume de la mobilisation, de sa percée médiatique ou de la satisfaction des revendications. On relève entre autres le cas d'habitants opposés à l'intensification du trafic dans leur rue qui, après avoir fait parvenir des pétitions (apparemment ignorées) à la mairie, ont attiré l'attention des journalistes locaux en bloquant tout simplement cette rue : «On habite dans la rue. On peut faire ça tous les soirs » (Le Progrès, $1^{\text {er }}$ juillet 2000). En somme, la durée d'un mouvement est favorable à l'ouverture de possibilités diverses, 
mais les premières actions s'accordent le plus souvent, par un effet de composition, à la reproduction de clichés.

Ainsi, l'étendue de ces réactions types est telle qu'elles emprisonnent l'ordinaire de l'action collective. 121 cas d'actions protestataires liés à l'activité professionnelle donnent lieu à des grèves. 13 fermetures de classes, à des occupations. 12 cas de mobilisations étudiantes ou lycéennes portant sur les motifs courants évoqués plus haut, à des manifestations. 10 mobilisations à l'échelle d'immeubles, cités ou quartiers, à des pétitions. 13 à des échelles allant de la commune au département, à des manifestations. 8 cas d'actions impliquant des partis politiques et/ou dont les motifs sont en rapport direct avec le champ politique sont des manifestations. En ajoutant les cas qui concernent les gardiens de prison et les routiers et en annulant les quelques recouvrements de catégories, 184 cas sur 286 donnent lieu aux réactions types liées à tel groupe et/ou situations mis en évidence dans l'article. Autrement dit, les réactions collectives types apparaissent dans près de deux tiers des cas relevés dans le corpus. Pour les raisons évoquées en introduction, cette proportion n'est pas représentative des actions protestataires françaises dans leur ensemble. Mais elle peut donner une première idée de la pression monumentale exercée sur chaque cas non par un répertoire limité mais par un seul mode d'action collective donné. Cela ne signifie pas que chaque situation ou déclencheur type soit une garantie de la réalisation de ce mode d'action : il est courant qu'aucune action collective n'ait lieu, aspect invisibilisé par mon matériau. Mais cela laisse a minima penser que les sentiers menant de ces situations au déclenchement d'un mode d'action donné laissent peu de place au choix et à l'improvisation en matière de formes de l'action collective.

Plusieurs types de cas échappent cependant à la force des réactions types comme ceux, sur lesquels on ne s'est pas arrêtés jusqu'ici, qui concernent des groupes partageant des logiques de distinction mais aussi d'emprunts multiples vis-à-vis des formes traditionnelles de l'action protestataires. On pense en particulier aux mouvements de groupes réputés « de droite » : artisans, commerçants, médecins libéraux, patrons d'exploitations agricoles, dont la position relativement centrale dans l'ACM (zone 4) s'explique en grande partie par le fait que l'on trouve chez eux des manifestations, mises en scènes, blocages routiers et saccages collectifs sans que l'un de ces modes ne domine nettement (Lemieux, 2018 ; Martin, 2014). Le fait que le mouvement des « gilets jaunes » soit significativement constitué de participants et leaders locaux non habitués du pavé et non identifiés à la gauche (Bedock et al., 2018) constitue à ce titre un des ressorts des quelques innovations ou techniques originales qui y ont pris place.

D'autres cas, auxquels on a fait allusion plus haut, offrent aussi des marges de manœuvre assez importantes sur les formes de l'action : ceux dont les différentes caractéristiques composent des mélanges relativement originaux qui ne permettent pas la reconnaissance certaine d'une situation de mobilisation type. Ou encore les innombrables situations qui ne sont pas associées à une réaction type connue et fortement institutionnalisée, qui se soldent le plus souvent par une absence de mobilisation mais 
dont les possibilités de construction apparaissent relativement ouvertes aux potentiels entrepreneurs de mobilisations. Les chercheurs s'intéressant aux répertoires de l'action collective ont d'ailleurs mis en avant le rôle des successions d'événements perçues comme inédites dans la réinvention des matrices et techniques de mobilisation (Cadiou, Dechézelles, 2007 ; McAdam, 1983 ; Offerlé, 2008 ; Sewell, 1996). C’est par ces cas que sont le plus susceptibles d'émerger de nouveaux types de scénarios de mobilisations, comme pour la bavure et l'émeute dans les années 1980-1990, ou plus récemment l'extension et les transformations des recours à la pétition rendue possible par les sites de pétitions en ligne (Boure, Bousquet, 2010).

\section{Bibliographie}

Bedock C., Bernard de Raymond A., Della Sudda M., Grémion T., Reungoat E., Schnatterer T. (2018), "Gilets jaunes" : une enquête pionnière sur "la révolte des revenus modestes" », Le Monde, 12 décembre, p. 22-23.

Berger P., Luckmann T. (2006 [1966]), La construction sociale de la réalité, Paris, Armand Colin (« Individu et société »).

Boure R., Bousquet F. (2010), « Enjeux, jeux et usages d’une pétition politique en ligne. "La pétition Vauzelle” », Réseaux, vol. 164, n 6, p.127-159.

Cadiou S., Dechézelles S. (2007), « La problématique de l'émergence pour l'étude des mobilisations collectives : pistes et repères ", in S. CAdiou, S. Dechézelles, A. Roger (dir.), Passer à l'action : les mobilisations émergentes, Paris, L'Harmattan (« Logiques politiques »), p. 11-48.

Contamin J.-G. (2009), « Pétition », in O. Fillieule, L. Mathieu, C. Péchu (dir.), Dictionnaire des mouvements sociaux, Paris, Presses de Sciences Po ("Académique. Sociétés en mouvement »), p. 319-320.

Courty G. (1993), " Barrer, filtrer, encombrer. Les routiers et l'art de retenir ses semblables », Culture er conflits, vol. 12, p. 143-168.

Fillieule O. (2008), « On n’y voit rien. Le recours aux sources de presse pour l'analyse des mobilisations protestataires », in P. Favre, O. Fillieule, F. Jobard (dir.), L'atelier du politiste. Théories, actions, représentations, Paris, La Découverte (« Recherches »), p. 215-240.

Fillieule O. (2010), « Tombeau pour Charles Tilly : répertoires, performances et stratégies d'action », in O. Fillieule, E. Agrikoliansky, I. Sommier (dir.), Penser les mouvements sociaux. Conflits sociaux et contestations dans les sociétés contemporaines, Paris, La Découverte (« Recherches »), p. 77-99.

Le Mazier J. (2015), "Pas de mouvement sans AG ». Les conditions d'appropriation de l'assemblée générale dans les mobilisations étudiantes en France (2006-2010), Thèse de doctorat en science politique, Paris, Université Paris 1 Panthéon-Sorbonne. 
Lemieux C. (2018), « Paradoxe de la modernisation. Le productivisme agricole et ses critiques (Bretagne, années 1990-2010) », Politix, vol. 123, nº 3, p. 115-144.

Martin J.-P. (2014), « Des paysans contestataires et novateurs (années 1960 - début du XXIe siècle) », in M. Pigenet, D. Tartakowsky (dir.), Histoire des mouvements sociaux en France. De 1814 à nos jours, Paris, La Découverte (" Poche/Sciences humaines et sociales »), p. 464-474.

Martin A. W., McCarthy J. D., Walker E. T. (2008), « Confronting the State, the Corporation, and the Academy: The Influence of Institutional Targets on Social Movement Repertoires », American Journal of Sociology, vol. 114, n 1, p. 35-76.

McAdam D. (1983), "Tactical Innovation and the Pace of Insurgency ", American Sociological Review, vol. 48, n 6, p. 735-754.

McAdam D. (1995), «Initiator and Spin-Off Movements: Diffusion Processes in Protest Cycle », in M. Traugott (dir.), Repertoires and Cycles of Collective Action, Durham/ Londres, Duke University Press, p. 217-239.

MоттA A. (2014), « Mépris et répression de la prise de parole en public. Construction d'une domination symbolique profane dans une copropriété et dénonciation publique », Participations, vol. 9, n 2, p. 71-95.

Moтta A. (2016), « La bavure et l'émeute. Genèse d'un signe déclencheur type dans le Rhône (1979-2000) », Revue française de science politique, vol. 66, nº 6, p. 937-961.

MotтA A. (2019), J-1. Sociologie des déclenchements d'actions protestataires, thèse de doctorat en science politique, Paris, Université Paris 1 Panthéon-Sorbonne.

Oberschall A. (1978), « Une théorie sociologique de la mobilisation », in P. Birnbaum, F. Chazel (dir.), Sociologie politique. Textes, Paris, Armand Colin (« Collection U2 »), p. 231-241.

OfFERLÉ M. (2008), « Retour critique sur les répertoires de l'action collective (XVIIIeXXIe siècles) », Politix, vol. 81, nº 1, p. 181-202.

RÉmy É. (1995), « Apprivoiser la technique. Débat public autour d'une ligne à haute tension », Politix, vol. 31, n³, p. 136-144.

Schelling T. (1986), Stratégie du conflit, Paris, Presses universitaires de France (« Perspectives internationales »).

Sewell W. (1996), " Historical Events as Transformation of Structures: Inventing Revolution at the Bastille», Theory and Society, vol. 25, n 6, p. 841-881.

Snow D., Zurcher L., Ekland-Olson S. (1980), « Social Networks and Social Movements: A Microstructural Approach to Differential Recruitment ", American Sociological Review, vol. 45, n 5, p. 787-801.

TaRTakowsky D. (2005), La part du rêve. Histoire du 1er mai en France, Paris, Hachette.

Tiluy C. (1978), From Mobilization to Revolution, Reading, Addison-Wesley. 
Tilly C. (2008), Contentious Performances, Cambridge, Cambridge University Press («Cambridge studies in contentious politics »). 